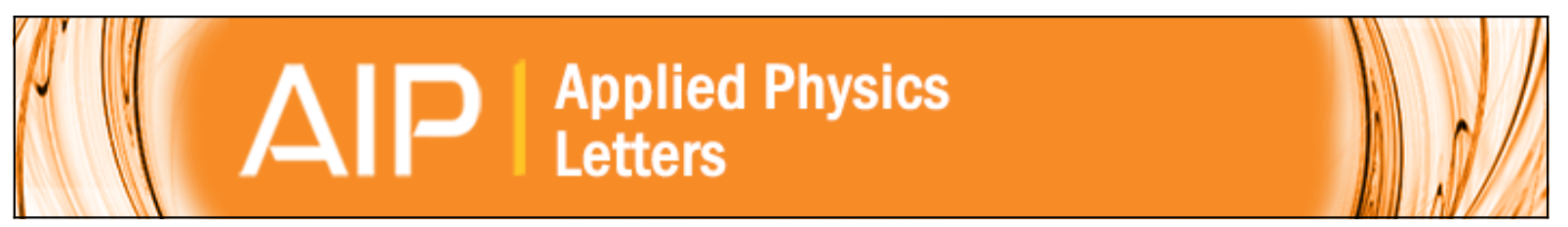

\title{
Temperature-dependent exciton-related transition energies mediated by carrier concentrations in unintentionally Al-doped ZnO films
}

Kun Tang, Shulin Gu, Jiandong Ye, Shimin Huang, Ran Gu, Shunming Zhu, Rong Zhang, Yi Shi, and Youdou Zheng

Citation: Applied Physics Letters 102, 221905 (2013); doi: 10.1063/1.4809669

View online: http://dx.doi.org/10.1063/1.4809669

View Table of Contents: http://scitation.aip.org/content/aip/journal/apl/102/22?ver=pdfcov

Published by the AIP Publishing

\section{Articles you may be interested in}

Experimental observation on the Fermi level shift in polycrystalline Al-doped $\mathrm{ZnO}$ films

J. Appl. Phys. 112, 013718 (2012); 10.1063/1.4733969

Growth morphology and electrical/optical properties of Al-doped $\mathrm{ZnO}$ thin films grown by atomic layer deposition J. Vac. Sci. Technol. A 30, 021202 (2012); 10.1116/1.3687939

Ga-related photoluminescence lines in Ga-doped $\mathrm{ZnO}$ grown by plasma-assisted molecular-beam epitaxy Appl. Phys. Lett. 94, 072101 (2009); 10.1063/1.3080204

Carrier concentration dependence of band gap shift in $\mathrm{n}$-type $\mathrm{ZnO}$ :Al films

J. Appl. Phys. 101, 083705 (2007); 10.1063/1.2721374

Temperature-dependent photoluminescence of quasialigned Al-doped $\mathrm{ZnO}$ nanorods

Appl. Phys. Lett. 90, 023104 (2007); 10.1063/1.2429906

\section{$\stackrel{A}{A} P$ Re-register for Table of Content Alerts}




\title{
Temperature-dependent exciton-related transition energies mediated by carrier concentrations in unintentionally Al-doped ZnO films
}

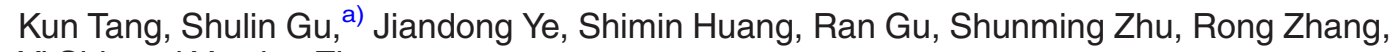 \\ Yi Shi, and Youdou Zheng \\ Nanjing National Laboratory of Microstructures and School of Electronic Science and Engineering, \\ Nanjing University, Nanjing 210093, People's Republic of China
}

(Received 25 March 2013; accepted 11 May 2013; published online 4 June 2013)

\begin{abstract}
The authors reported on a carrier-concentration mediation of exciton-related radiative transition energies in $\mathrm{Al}$-doped $\mathrm{ZnO}$ films utilizing temperature-dependent (TD) photoluminescence and TD Hall-effect characterizations. The transition energies of free and donor bound excitons consistently change with the measured TD carrier concentrations. Such a carrier-concentration mediation effect can be well described from the view of heavy-doping-induced free-carrier screening and band gap renormalization effects. This study gives an important development to the currently known optical properties of $\mathrm{ZnO}$ materials. (C) 2013 AIP Publishing LLC. [http://dx.doi.org/10.1063/1.4809669]
\end{abstract}

The characteristic transition lines of intrinsic $\mathrm{ZnO}$ bulk materials $\left(I_{0}-I_{11}\right.$ in prior literatures) have been well established by the low-temperature (LT) and temperaturedependent (TD) photoluminescence (PL) measurements. ${ }^{1}$ It is still reasonable when applying all the assignments to nominally undoped $\mathrm{ZnO}$ films in spite that the carrier concentrations are much higher $\left(\sim 10^{16} \mathrm{~cm}^{-3}\right)$ than those in the $\mathrm{ZnO}$ bulks $\left(\sim 10^{14} \mathrm{~cm}^{-3}\right)$. In case of the undoped films, the high doping effects (HDEs) [e.g., the free-carrier screening (FCS) effect, the Burstein-Moss (BM) effect, and the band gap renormalization (BGR) effect] are almost negligible. However, these effects would become more prominent when the carrier concentration goes higher. The FCS effect will modify a spherical Coulomb potential, resulting in a reduced exciton binding energy and then a blueshift of the optical transition associated with both free and bound excitons. ${ }^{2}$ In addition, the BM effect, aka the Fermi-level band filling effect, and the BGR effect will show a competitive effect on shifting the transition energies of the excitons. ${ }^{3}$ Consequently, one should be immensely careful when trying to assign exciton-related transition lines in the impuritydoped $\mathrm{ZnO}$ films, especially based on the TD transition energy positions. However, researchers seldom paid attention to this issue. In fact, the reported binding energies of impurities varied a lot [e.g., $E_{\mathrm{D}}(\mathrm{H})=46-57 \mathrm{meV}$ and $\left.\left.E_{\mathrm{D}}(\mathrm{Al})=51-55 \mathrm{meV}\right)\right]^{4,5}$ Despite that the diversity of the samples due to different apparatuses and growth conditions should be responsible for the variation, the neglect of taking the HDEs into account might also be a partial reason.

In order to investigate the influence of the HDEs on modifying the exciton-related transition energies in impurity-doped $\mathrm{ZnO}$ films, samples have to be elaborately selected. If the samples are heavily doped, although the HDEs are obvious, the fine structures of the PL transition lines can hardly be resolved at a low temperature due to a high doping concentration. ${ }^{6}$ This is why most of the current researching works on the HDEs of transparent conductive

\footnotetext{
${ }^{\text {a) }}$ Author to whom correspondence should be addressed. Electronic mail: slgu@nju.edu.cn
}

oxides (TCOs) are based on room-temperature (RT) optical characterizations (e.g., PL and absorption spectra). ${ }^{7-11}$ However, if the doping concentration of the samples is too low, no visible HDEs could be detected. In this letter, we have artificially modified the electron carrier concentrations of three $\mathrm{ZnO}$ films adequately doped with $\mathrm{Al}$ during the TD measurement and tracked the trends of the exciton-related transition lines as well. Importantly, this experimental design is not applicable to the heavily doped case because in degenerate materials, the carrier concentration does not change obviously with temperature ${ }^{12}$ and the positions of the transition lines are nearly impossible to be extracted accurately due to the ambiguously merged PL peak components. As a result, this study may provide some substantial results on the carrier-concentration-mediated TD modifications of transitions related to bound and free excitons (FXs) not only on the RT band gap modification which had been extensively studied via the HDEs of the heavily doped TCOs.

Actually, the $\mathrm{Al}$ dopants in our $\mathrm{ZnO}$ samples discussed in this letter are not deliberately incorporated. The primary target of this batch is to investigate the growth of an undoped $\mathrm{ZnO}$ epilayer with a high crystalline quality on a sapphire substrate. However, the post-growth Hall-effect characterization showed that all the samples have electron carrier concentrations of $10^{18}-10^{19} \mathrm{~cm}^{-3}$, which were far from the original anticipation. Through deep investigation, ${ }^{13}$ the interdiffusion of $\mathrm{Al}$ atoms from the sapphire substrate has been recognized as the origin of the electron carrier concentration and three pieces of "unintentionally" Al-doped $\mathrm{ZnO}$ (AZO) films are consequently obtained. All samples were grown by an AIXTRON metal-organic chemical vapour deposition (MOCVD) system exclusively designed for the $\mathrm{ZnO}$ material with vertical gas flows through a showerhead configuration. A ZnO film was first grown on a sapphire substrate at $445^{\circ} \mathrm{C}$ using dimethylzinc $(\mathrm{DMZn})$ and tert-butanol $(\mathrm{t}-\mathrm{BuOH})$ as precursors, and the substrate temperature was linearly increased from $445^{\circ} \mathrm{C}$ to $1050^{\circ} \mathrm{C}$ within $5 \mathrm{~min}$. The resulted sample is denoted as sample A with a thickness of $379 \mathrm{~nm}$. Subsequently, a $21-\mathrm{nm}$ thin $\mathrm{ZnO}$ layer was grown at $1050^{\circ} \mathrm{C}$ using DMZn and $\mathrm{N}_{2} \mathrm{O}$ as precursors with a low VI/II ratio on 
the ready-made sample A. The resulted 400-nm-thick $\mathrm{ZnO}$ film is denoted as sample B. Finally, sample C has a thickness of $3.27 \mu \mathrm{m}$, which is resulted from a 2-h-long growth on the ready-made sample $\mathrm{B}$ with the same growth condition but a modified VI/II ratio.

One thing should be recalled here that the full widths at half maximum (FWHM) of the $\mathrm{ZnO}$ (002) x-ray rocking curve (XRC) are 727, 623, and 266 arc sec for samples A, B, and $\mathrm{C}$, respectively, indicative of high crystalline quality of $\mathrm{ZnO}$ films grown by a MOCVD technique. Moreover, based on the atomic force microscopic observation, the grain sizes of the samples are larger (all $>1 \mu \mathrm{m}$ ) enough to make the scattering mechanism much simpler. Small XRC FWHMs as well as large grains guaranteed the authenticity of the TD Hall-effect and TD PL measurement, which is a precondition for a further analysis. Two helium cryogenic chambers were employed for the electrical and optical measurement, respectively. In the 1st chamber, four tungsten probes were placed on the four indium pads sticked on the corners of a $5 \mathrm{~mm} \times 5 \mathrm{~mm}$ sliced sample to ensure the resistivity and Hall coefficient measurement under a van der Pauw's configuration. On the side of the 2nd chamber, a window was opened for a 325-nm helium-cadmium laser to go through and excite the samples. The measuring temperature from $15 \mathrm{~K}$ to $300 \mathrm{~K}$ was controlled by two Lakeshore (Model 336) temperature controllers.

Figure 1 shows the overview of the TD PL spectra of all the samples recorded at the temperatures ranging from $15 \mathrm{~K}$ to $300 \mathrm{~K}$ and the assignments for each component. Most of the assignments are conspicuous based on the experience in analysing the TD PL spectra of $\mathrm{ZnO}$. In a prudential way, however, we shall still show the evidence of some of the assignments in the following parts of this letter. In Fig. 1, the thermal quenching of the transition lines from excitons bound to neutral donors $\left(\mathrm{D}^{0} \mathrm{X}\right)$ and the emergence of the corresponding FXs transition can be clearly seen for samples A and $\mathrm{C}$ indicative of their good optical qualities. For sample $\mathrm{B}$, the demarcation is not as distinctive as samples $\mathrm{A}$ and $\mathrm{C}$ due to its relatively high carrier concentration at LT range. ${ }^{6}$ Accordingly, in the following discussion, the positions of the components are acquired via peak fittings.

The band gap of $\mathrm{ZnO}$ between conduction band minimum and the A-valence subband maximum $\left(E_{\mathrm{gA}}\right)$ is $3.437 \mathrm{eV}$ at $0 \mathrm{~K}$, and the temperature evolutionary trend of the $E_{\mathrm{gA}}$ is described by the well-known Varshni's empirical relation. $\left(\alpha=5.05 \times 10^{-4} \mathrm{eV} / \mathrm{K} \text { and } \beta=900 \mathrm{~K}\right)^{1}$ As a result, if no $\mathrm{HDE}$ is considered, the energy positions of the FX $\left(E_{\mathrm{FX} 0}\right)$ should strictly follow the Varshni's relation. In order to see whether there is any difference between the FX positions of the samples $\left(E_{\mathrm{FX}}\right)$ and the $E_{\mathrm{FX} 0}$, the $E_{\mathrm{FX}}$ values of all the temperature points are then extracted. Figure 2 plots the values of the $\left|E_{\mathrm{FX}}-E_{\mathrm{FX} 0}\right|$ for all the samples (scattered squares). It can be observed from the figure that the $\left|E_{\mathrm{FX}}-E_{\mathrm{FXo}}\right|$ varies with temperature and does not equal zero at any point implying that the $E_{\mathrm{FX} 0}$ values are not exactly coincident with the $E_{\mathrm{FX}}$ ones.

In addition, it is noticed that the evolutionary trends of the $\left|E_{\mathrm{FX}}-E_{\mathrm{FX} 0}\right|$ are in perfect agreement with those of the electron carrier concentration as shown in the inset of Fig. 2. The concentrations do not monotonously increase with
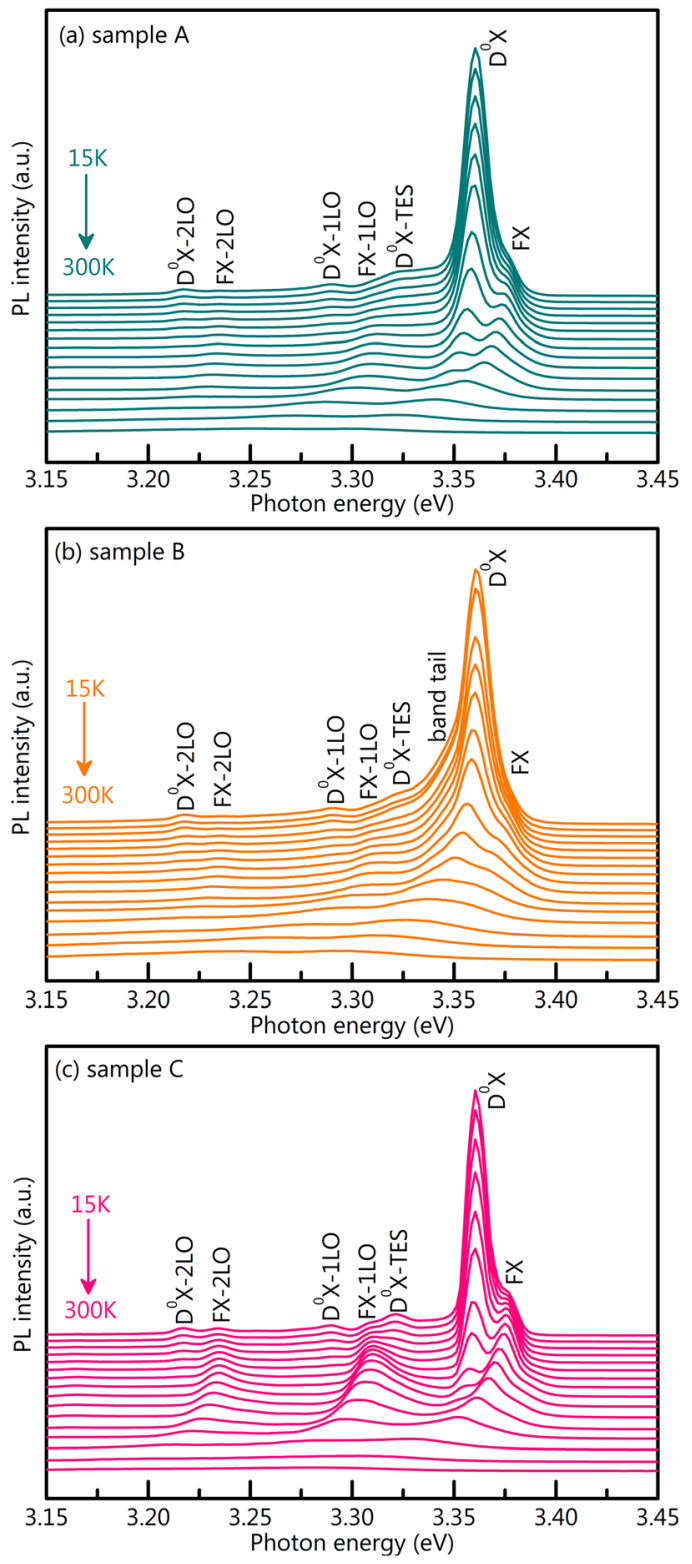

FIG. 1. TD PL spectra recorded from $15 \mathrm{~K}$ to $300 \mathrm{~K}$ of (a) a $\mathrm{ZnO}$ film grown at $445^{\circ} \mathrm{C}$ using $\mathrm{DMZn}$ and $\mathrm{t}-\mathrm{BuOH}$ as precursors and annealed during a ramp of temperature from $445^{\circ} \mathrm{C}$ to $1050^{\circ} \mathrm{C}$ for $5 \mathrm{~min}$ (sample A), (b) a thin $\mathrm{ZnO}$ layer subsequently grown on the sample $\mathrm{A}$ at $1050^{\circ} \mathrm{C}$ for $10 \mathrm{~min}$ using DMZn and $\mathrm{N}_{2} \mathrm{O}$ as precursors (sample B), and (c) a thick ZnO film subsequently grown on the sample $\mathrm{B}$ at $1050{ }^{\circ} \mathrm{C}$ for $2 \mathrm{~h}$ using DMZn and $\mathrm{N}_{2} \mathrm{O}$ as precursors (sample C).

temperature indicative of non-uniformity in electrical properties along the epi-direction of the films. ${ }^{14}$ The increase of the concentrations at lower temperatures $(<150 \mathrm{~K})$ is usually ascribed to highly conductive layer(s) formed on the film surface and/or on the interfaces (e.g., a buffer layer/substrate interface). This trend is also found in other semiconductors due to the impurities-band effect induced by doping at a moderate or even a bit higher level. ${ }^{15}$ In order to obtain the electrical properties in the bulk, Look $^{14}$ had developed a two-layer Hall-effect model, which could subtract the contribution from the highly conductive layer(s). Following this concept, the carrier concentrations of the samples had been corrected in Reference 13. However, the consensus has been obtained on the raw data, not the corrected ones, which 


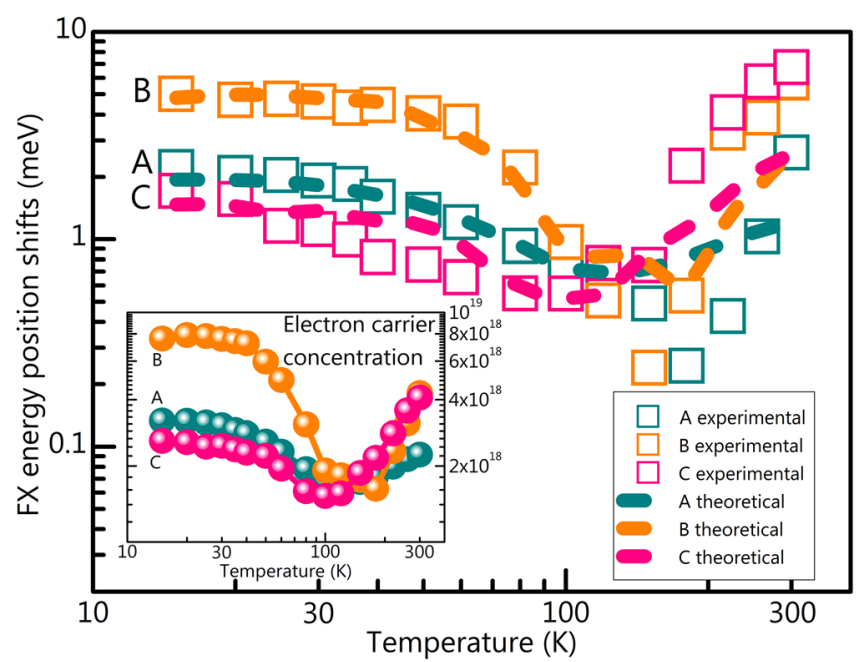

FIG. 2. TD FX energy position shifts (squares) and the calculated values by Eq. (3) (dashed lines). The inset plots the TD carrier concentration of all the samples.

strongly indicates the highly conductive layer may give a contribution to the measured TD PL spectra.

Since all the HDEs are closely linked to the carrier concentration, wherever it comes from (surface or bulk), and upon the consideration of the abovementioned experimental consistency, it can be concluded that HDEs cannot be ignored in these samples. For the BM effect, the electrons in the filled bands can still irradiate under the laser excitation, so the optical band gap variation from the effect should be characterized by the absorption edge shift instead of PL spectra. ${ }^{16}$ Moreover, the lowest states in the conduction band begin to be filled with electrons only when the carrier concentration $n$ increases above the conduction band density of states (DOS): $N_{C}=2\left(2 \pi m^{*} k T\right)^{3 / 2} / h^{3}$, which is around $3.6 \times 10^{18} \mathrm{~cm}^{-3}$ for $\mathrm{ZnO} .{ }^{17}$ Since the range of carrier densities is from $1 \times 10^{18} \mathrm{~cm}^{-3}$ to $8 \times 10^{18} \mathrm{~cm}^{-3}$ for our samples as shown in the inset of Fig. 2, the blueshifts induced by the BM effect should not be conspicuous for all the samples. For the FCS effect, noticing that we have mentioned the effect in the 1st paragraph, the influence of this effect results in a decrease in the binding energy of both free and bound excitons. $^{2}$ More importantly, a substantial screening will occur when the Debye length $\left(\sim \varepsilon k T / e^{2} n_{\text {eff }}\right)$ and the Bohr radius $\left(\sim 0.529 \varepsilon / \mathrm{m}^{*}\right)$ are comparable, ${ }^{2}$ where $n_{\text {eff }}$ denotes the concentration of the electron carriers effectively participating in the screening process. Reynolds et al. calculated the $n_{\text {eff }}$ to be $\sim 10^{16} \mathrm{~cm}^{-3}$ for $\mathrm{ZnO}{ }^{2}$ Hence it is expected that FCS effect will be prominent in our samples. As a result, the HDEs are modelled as a combined effect of a blueshift from the FCS effect and a redshift from the BGR effect. ${ }^{16}$

An empirical equation expressing the FCS effect of donor activation energy has been given by ${ }^{2}$

$$
E_{\mathrm{D}}=E_{\mathrm{D} 0}-\alpha n^{1 / 3}
$$

where $E_{\mathrm{D}}$ and $E_{\mathrm{D} 0}$ denote the screened and unscreened donor activation energies, and $\alpha$ is a parameter. Moreover, it is also pointed out that the free exciton binding energy would be screened very similar to that of the donor activation energy. ${ }^{2}$ Therefore, it is rational to introduce a term of $\Delta E^{\text {FCS }}$
$=K n^{1 / 3}$, which describes the blueshift from the FCS effect. For modelling the BGR effect, an expression suggested in Ref. 17 is adopted

$$
\Delta E^{\mathrm{BGR}}=A n^{1 / 3}+B n^{1 / 4}+C n^{1 / 2} .
$$

In a simplified model, $A$ and $B$ are constants and $C$ is an adjustable parameter related to a ratio of majority- divided by minority-carrier density-of-state effective mass $\left(m_{\mathrm{maj}}^{*} / m_{\mathrm{min}}^{*}\right)$. This model is much more accurate compared to the wellacknowledged $n^{1 / 3}$ dependence ${ }^{17}$ because it considers all the possible interactions like electron-electron, electron-ion, hole-hole, and hole-ion interactions while the $n^{1 / 3}$ dependence is based on a weakly interacting electron-gas model, which only takes the electron-electron interactions into account. ${ }^{17}$ Finally, the energy position of the FX transition is given by

$$
E_{\mathrm{FX}}=E_{\mathrm{FX} 0}+\Delta E^{\mathrm{FCS}}-\Delta E^{\mathrm{BGR}}
$$

The dashed lines in Fig. 2 are the simulated results of the $\left|E_{\mathrm{FX}}-E_{\mathrm{FX} 0}\right|$ based on Eq. (3) with $C=7.76 \times 10^{-12} \mathrm{eV} \mathrm{cm}^{3 / 2}$ and $K=2.11 \times 10^{-8} \mathrm{eV} \mathrm{cm}$. The calculation accords well with the experimental data points below $120 \mathrm{~K}$ indicative of the validity of the developed formulation. However, above $150 \mathrm{~K}$, the experimental values are generally larger than those of the theoretical prediction. It is not due to that the model is not applicable in the relatively high-temperature range. The redshifts of the FX transitions are larger compared to the Varshni's prediction at temperatures above $160 \mathrm{~K}$ even in the intrinsic $\mathrm{ZnO}$ bulk materials. ${ }^{18}$ It could be understood like this: as the measuring temperature is increased, the FX and its phonon replicas are coupled with the line broadening of each of these peaks, which will definitely hinder an accurate determination of the peak position of the FX transition.

To understand the high consensus obtained on the redshifts of the FX transitions and the measured carrier concentrations, we then move to study the doping concentrations of the samples. Secondary ion mass spectrometry (SIMS) measurements have revealed a high distribution of the $\mathrm{Al}$ elements both near the surface and the $\mathrm{ZnO} /$ sapphire interface compared to the bulk region for all the samples. (See Ref. 13 for the depth profile of the Al elements.) The actual atomic concentration of $\mathrm{Al}$ is not available due to the lack of a standard AZO sample with accurately known concentration of $\mathrm{Al}$. However, since the $\mathrm{Al}$ ion counts are in direct proportion to the atomic concentration of $\mathrm{Al}$, the $\mathrm{Al}$ ion counts could be definitely used as a representative for the atomic concentration of $\mathrm{Al}$. As the substituting $\mathrm{Al}$ is commonly regarded as an active shallow donors in $\mathrm{ZnO}$, the accumulation of these elements would lead to the formation of a top surface conductive layer as well as a highly conductive layer on the interface. The measured Hall-effect results are actually the averaged values of the carrier concentrations over the total thickness of the film, including the top conductive layer, the intrinsic bulk layer, and the bottom conductive layer. The highly conductive layers existing on the surface and the interface should be mainly responsible for the degenerate features observed in the LT region of the TD Halleffect measurement for all the samples. 
The penetration depth of a $325-\mathrm{nm}$ exciting laser is about $60 \mathrm{~nm},{ }^{19}$ which means that the excited carriers are mostly located within the penetration depth as well as the diffusion length of the minority carriers. As the diffusion length of the minority carriers (holes) in a bulk $\mathrm{ZnO}$ was measured to be longer than $400 \mathrm{~nm},{ }^{20}$ it is reasonable for us to assume a similar diffusion length of about $300-400 \mathrm{~nm}$ for the MOCVD-grown $\mathrm{ZnO}$ samples. In this case, the epilayers with the thicknesses of $379 \mathrm{~nm}$ and $400 \mathrm{~nm}$ for samples A and B should totally contribute to the PL emission. However, for sample C, only the surface layer within $\sim 400$ $\mathrm{nm}$, i.e., a small part of the epilayer (total thickness of $3.27 \mu \mathrm{m}$ ), can contribute. It is consequently easy to understand why high consensus can be reached between the redshift of the FX transitions and the measured carrier concentrations at the LT regime for samples A and B, but a small deviation is obviously found for sample $\mathrm{C}$ as shown in Fig. 2.

After modelling the FX transition energies, it is natural to move forward to the dominant $\mathrm{D}^{0} \mathrm{X}$ transitions. As mentioned before, under the screened condition, the energy position of the $\mathrm{D}^{0} \mathrm{X}\left(E_{\mathrm{D}^{0} \mathrm{X}}\right)$ should be determined by subtracting the localization energy of the $\mathrm{D}^{0} \mathrm{X}$ from the $E_{\mathrm{FX}}$. Since the donor activation energy is screened and according to the Haynes rule, the localization energy should also be screened. ${ }^{2}$ Therefore, it would be interesting to see how the $\mathrm{D}^{0} \mathrm{X}$ transition energies change with the measuring temperatures, i.e., the carrier concentrations. We again utilize the peak deconvoluting method to extract the peak positions of the $\mathrm{D}^{0} \mathrm{X}$ for all the samples. It definitely shows some differences compared to the trends of the FX. Taking the $15 \mathrm{~K}$ point as an example, over $3 \mathrm{meV}$ energy difference of the $E_{\mathrm{FX}}$ positions is observed for different samples as shown in Fig. 2. However, the positions of the $\mathrm{D}^{0} \mathrm{X}$ are almost invariable and all located at $3.3607 \mathrm{eV}$ which suggest that the localization energies of the samples are not exactly same. Consequently, the $\mathrm{D}^{0}$ activation energy shifts of all the samples from $15 \mathrm{~K}$ to $80 \mathrm{~K}$ have been calculated using the following equation:

$$
\Delta E_{\mathrm{D}}=55 \mathrm{meV}-\left(E_{\mathrm{FX}}-E_{\mathrm{D}^{0} \mathrm{X}}\right) / 0.33,
$$

where 0.33 is the Haynes factor for $n$-type $\mathrm{ZnO}^{4}$ and $55 \mathrm{meV}$ is the generally accepted $\mathrm{D}^{0}$ activation energy of $\mathrm{Al}_{\mathrm{Zn}}$ donors in $\mathrm{ZnO}\left(E_{\mathrm{D} 0}\right) .{ }^{1}$ The calculated values are shown in Fig. 3 versus their respective $n^{1 / 3}$ as the values for the $x$ coordinate. A perfect linear relation between $\Delta E_{\mathrm{D}}$ and $n^{1 / 3}$ could be seen, which demonstrates that the blueshifts of the $\mathrm{D}^{0} \mathrm{X}$ energies are mainly ascribed to the FCS effect. The fitted slope is $\sim 1.60 \times 10^{-8} \mathrm{eV} \mathrm{cm}$, consistent with a reported value for $\mathrm{ZnO}\left(1.2 \times 10^{-8} \mathrm{eV} \mathrm{cm}\right){ }^{2}$ However, it is noted that the extrapolation of the fitted line does not pass across the origin, which is somewhat inconsistent with Eq. (1). In our opinion, it could be partially due to the Haynes factor we used in the calculation since it is not a constant for all the $\mathrm{ZnO}$ materials (the reported values range from 0.3 to 0.36 ). ${ }^{4}$ In addition, the $E_{\mathrm{D}}$ value of the unscreened $\mathrm{D}^{0} \mathrm{X}\left(E_{\mathrm{D} 0}\right)$ may not be accurate because the number of $55 \mathrm{meV}$ is calculated from the energy distance between the $\mathrm{D}^{0} \mathrm{X}$ and its twoelectron satellite (TES) in an AZO based on LT-PL

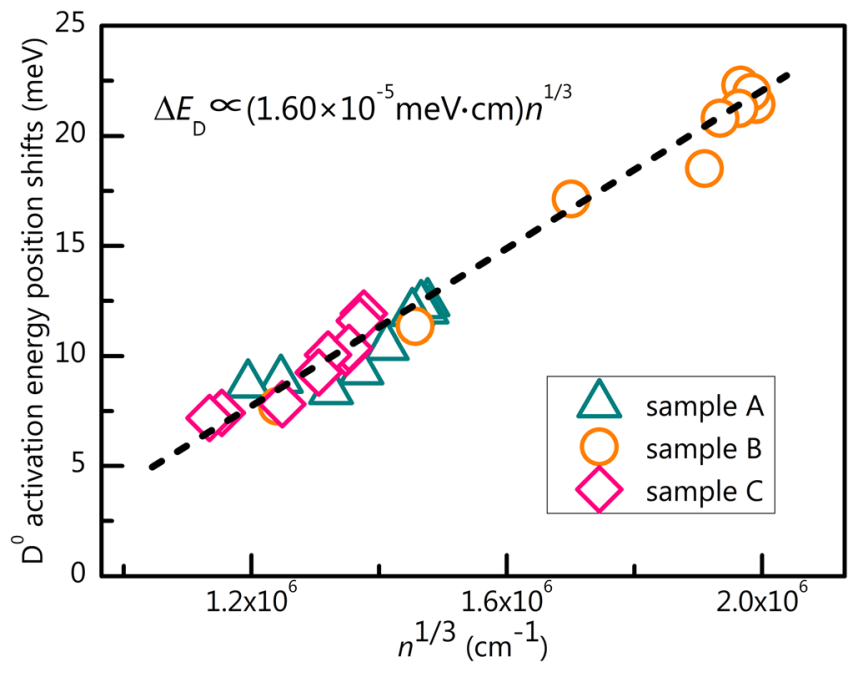

FIG. 3. TD activation energies of $\mathrm{D}^{0}$ as a function of $n^{1 / 3}$ from $15 \mathrm{~K}$ to $80 \mathrm{~K}$ and their linear fit (dashed line).

technique. ${ }^{1}$ Since the neutral donor $\mathrm{D}^{0}$ is screened, the energy of its excited states ( $2 p$ ) should be different compared to that in the unscreened case, which means the activation energy of $55 \mathrm{meV}$ is also calculated in a screened system with a non-zero carrier concentration. As a result, the intercept of the line at $\Delta E_{\mathrm{D}}=0$ corresponds to a finite carrier concentration as shown in Fig. 3.

The blueshifts of the TES transitions with increasing the carrier concentration are proven in the $15 \mathrm{~K}$ PL spectra as identified in Fig. 4. The values of the $\left(E_{\mathrm{D}^{0} \mathrm{X}}-E_{\mathrm{TES}}\right)$ are $36.94,31.73$, and $39.60 \mathrm{meV}$ for samples A-C, respectively. Considering the $\mathrm{D}^{0}$ activation energies calculated by Eq. (4), the ratios of $E_{D} /\left(E_{\mathrm{D}^{0} \mathrm{X}}-E_{\mathrm{TES}}\right)$ are $1.15,1.03$, and 1.09 for samples A-C, respectively. The empirical relation $E_{D}$ $=$ const. $\times\left(E_{\mathrm{D}^{0} \mathrm{X}}-E_{\mathrm{TES}}\right)$ is at least tenable but the constant is smaller than $4 / 3,{ }^{1}$ suggesting that the FCS effect on modifying the energy of the excited states of the neutral donor $\mathrm{D}^{0}$ is slight stronger than that of its activation energy. Furthermore, a band tail transition has been found with different intensities for the samples as marked by a

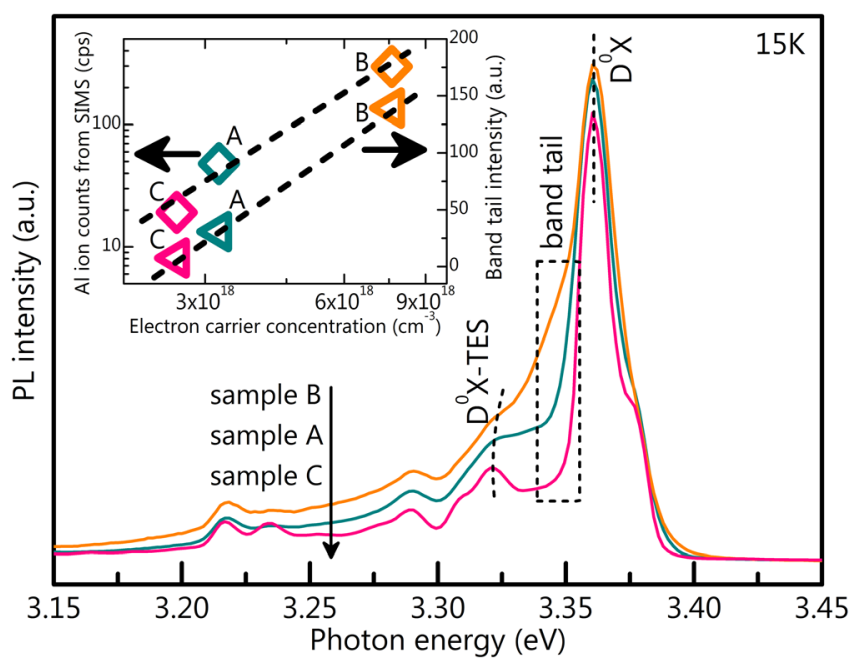

FIG. 4. $15 \mathrm{~K}$ PL spectra of all the samples. The inset shows the Al ion counts from SIMS and the integral intensity of the band tail components as a function of the carrier concentration for all the samples. 
rectangle in Fig. 4. He et al. once assigned this component to another bound exciton-related transition for their AZO nanorods, ${ }^{21}$ but they had not given an explicit origin to that transition there. Noticing that the trend of the intensities of this band tail shows strong correlation to the Al elements concentration as well as the electron carrier concentration as illustrated in the inset of Fig. 4. As a result, it is believed that the band tail should be related to the $\mathrm{Al}$ dopants. If it is a $\mathrm{D}^{0} \mathrm{X}$ transition and concerning $\mathrm{Al}$, apart from the substituting $\mathrm{Al}_{\mathrm{Zn}}$ donors, the $\mathrm{D}^{0}$ should be interstitial $\mathrm{Al}$ ion that acts as a trivalent donor. However, based on a recent theoretical study, they have eliminated the possibility of $\mathrm{Al}^{3+}$ ions as interstitial impurities. ${ }^{22}$ Accordingly, the band tail is ascribed to the recombination from the discrete energy levels and/or the continuous energy bands near the conduction band edge to the valence band maximum. The energy levels or bands are created by the band tailing effect due to a high doping concentration. ${ }^{23}$ When the doping concentration increases, the density of the randomly distributed charged impurities also increases resulting in a potential fluctuation in the vicinity of the conduction band edge, and the potential fluctuation would cause the band edge energy to vary spatially. ${ }^{23}$ There upon, there are always fluctuated band edges that are below the unperturbed one and the band tail states are created then. Since the Fermi-Dirac function is very close to 1 below the Fermi level when the temperature is close to absolute zero, the radiative recombination related to the band tail states just below the conduction band edge could be evidently captured in spite of relatively low DOS.

On the lower energy side to the band tail and the TES transition, only the $\mathrm{D}^{0} \mathrm{X}$ and $\mathrm{FX}$ related phonon replicas can be seen as Figs. 2 and 4 are attentively reviewed. One thing is deserved to be mentioned that the energy positions of the 1st and 2nd phonon replicas of the FX are not fully along the evolutionary trend of the FX as measuring temperature is changed. The energies of the replicas are given by ${ }^{18}$

$$
\begin{gathered}
E_{\mathrm{FX}-1 \mathrm{LO}}=E_{\mathrm{FX}}-\hbar \omega_{\mathrm{LO}}+\frac{3}{2} k T, \\
E_{\mathrm{FX}-2 \mathrm{LO}}=E_{\mathrm{FX}}-2 \hbar \omega_{\mathrm{LO}}+\frac{1}{2} k T,
\end{gathered}
$$

where the $\hbar \omega_{\mathrm{LO}}=72 \mathrm{meV}$ denotes the LO phonon energy. Because the free-electron-to-acceptor (FA) transition also follows the trends expressed by Eq. (5) or (6), ${ }^{21}$ one should be very cautious when assigning the FA transition based on the energy position evolution. However, in our samples, the components marked LO phonon replicas in Fig. 2 are undoubted as the intensity consistency between the FX and its phonon replicas is also checked. Therefore, it can be concluded that no acceptor-related recombination lines are detected.

In summary, TD PL and TD Hall-effect measurements have been utilized to investigate the TD modifications of the recombination lines related to both free and bound excitons in $\mathrm{ZnO}$ films of high crystalline qualities. Carrier concentration dependent energy shifts of the FX have been observed and modelled by taking into account the HDEs including the
FCS and BGR effects. The activation energy of the dominant $\mathrm{Al}_{\mathrm{Zn}}$ donors is also modulated by the carrier concentrations at different measuring temperature mainly due to the FCS effect, which results in modifications to the energy positions of both $\mathrm{D}^{0} \mathrm{X}$ and its TES. In addition, an obvious radiative transition related to band tail states corresponds to the high doping concentration. This study provides a direct evidence to the energy shift of the exciton-related transitions induced by the HDEs, which is previously underestimated or ignored by researchers. It may hence be an important supplement to the current knowledge of the PL properties of $\mathrm{ZnO}$ materials.

This research is supported by the State Key Program for Basic Research of China under Grant No. 2011CB302003, National Natural Science Foundation of China (Nos. 61025020, 60990312, and 61274058), Basic Research Program of Jiangsu Province (BK2011437), and the Priority Academic Program Development of Jiangsu Higher Education Institutions.

${ }^{1}$ B. K. Meyer, H. Alves, D. M. Hofmann, W. Kriegseis, D. Forster, F. Bertram, J. Christen, A. Hoffmann, M. Straßburg, M. Dworzak, U. Haboeck, and A. V. Rodina, Phys. Status Solidi B 241, 231 (2004).

${ }^{2}$ D. C. Reynolds, D. C. Look, and B. Jogai, J. Appl. Phys. 88, 5760 (2000).

${ }^{3}$ B. Singh, Z. A. Khan, I. Khan, and S. Ghosh, Appl. Phys. Lett. 97, 241903 (2010).

${ }^{4}$ H. Morkoç and Ü. Özgür, in Zinc Oxide: Fundamentals, Materials and Device Technology (Wiley, New York, 2009), p. 171.

${ }^{5}$ S. S. Lin, H. P. He, Z. Z. Ye, B. H. Zhao, and J. Y. Huang, J. Appl. Phy. 104, 114307 (2008).

${ }^{6}$ K. Tang, S. L. Gu, J. D. Ye, S. M. Huang, R. Gu, R. Zhang, and Y. D. Zheng, J. Appl. Phys. 112, 103534 (2012).

${ }^{7}$ Y. Kim, W. Lee, D.-R. Jung, J. Kim, S. Nam, H. Kim, and B. Park, Appl. Phys. Lett. 96, 171902 (2010).

${ }^{8}$ M. Bazzani, A. Neroni, A. Calzolari, and A. Catellani, Appl. Phys. Lett. 98, 121907 (2011).

${ }^{9}$ P. Banerjee, W.-J. Lee, K.-R. Bae, S. B. Lee, and G. W. Rubloff, J. Appl. Phys. 108, 043504 (2010).

${ }^{10}$ H. H.-C. Lai, T. Basheer, V. L. Kuznetsov, R. G. Egdell, R. M. J. Jacobs, M. Pepper, and P. P. Edwards, J. Appl. Phys. 112, 083708 (2012).

${ }^{11}$ W. F. Yang, Z. Y. Wu, Z. G. Liu, A. S. Pang, Y.-L. Tu, and Z. C. Feng, Thin Solid Films 519, 31 (2010).

${ }^{12}$ Z. Yang, D. C. Look, and J. L. Liu, Appl. Phys. Lett. 94, 072101 (2009).

${ }^{13}$ K. Tang, S. L. Gu, S. Z. Li, J. D. Ye, S. M. Zhu, H. Chen, J. G. Liu, R. Zhang, Y. Shi, and Y. D. Zheng, J. Vac. Sci. Technol. A 29, 03 A106 (2011).

${ }^{14}$ D. C. Look, J. Appl. Phys. 104, 063718 (2008).

${ }^{15}$ D. C. Look, in Electrical Characterization of GaAs Materials and Devices (Wiley, New York, 1989), p. 115.

${ }^{16}$ J. D. Ye, S. L. Gu, S. M. Zhu, S. M. Liu, Y. D. Zheng, R. Zhang, and Y. Shi, Appl. Phys. Lett. 86, 192111 (2005).

${ }^{17}$ J. G. Lu, S. Fujita, T. Kawaharamura, H. Nishinaka, Y. Kamada, T. Ohshima, Z. Z. Ye, Y. J. Zeng, Y. Z. Zhang, L. P. Zhu, H. P. He, and B. H. Zhao, J. Appl. Phys. 101, 083705 (2007).

${ }^{18}$ D. W. Hamby, D. A. Lucca, J. J. Klopfstein, and G. Cantwell, J. Appl. Phys. 93, 3214 (2003).

${ }^{19}$ H. Chen, S. L. Gu, J. G. Liu, J. D. Ye, K. Tang, S. M. Zhu, and Y. D. Zheng, Appl. Phys. Lett. 99, 211906 (2011).

${ }^{20}$ O. Lopatiuk, L. Chernyak, A. Osinsky, J. Q. Xie, and P. P. Chow, Appl. Phys. Lett. 87, 162103 (2005).

${ }^{21}$ H. P. He, H. P. Tang, Z. Z. Ye, L. P. Zhu, B. H. Zhao, L. Wang, and X. H. Li, Appl. Phys. Lett. 90, 023104 (2007).

${ }^{22}$ J.-T. Thienprasert, S. Rujirawat, W. Klysubun, J. N. Duenow, T. J. Coutts, S. B. Zhang, D. C. Look, and S. Limpijumnong, Phys. Rev. Lett. 110, 055502 (2013).

${ }^{23}$ C. H. Zang, J. F. Su, B. Wang, D. M. Zhang, and Y. S. Zhang, J. Lumin. 131, 1817 (2011). 